\title{
Algorithm and Application of Inverse Kinematics for 6-DOF Welding Robot Based on Screw Theory
}

\author{
Lei Hong ${ }^{1}$, Baosheng Wang ${ }^{2}$, Zhenqin $\mathrm{Xu}^{2}$ and Dongsheng $\mathrm{Lv}^{2}$ \\ ${ }^{1}$ School of Automotive and Rail Transit, Nanjing Institute of Technology, Nanjing, China \\ ${ }^{2}$ Research Department of Intelligent Manufacturing Equipment, Nanjing Institute of Technology, Nanjing, China
}

\begin{abstract}
In order to achieve efficient and reliable inverse kinematics for industrial robot, an inverse kinematics solution is proposed for common $6 \mathrm{DOF}$ industrial robots with the structure characteristics that can be described as "the last three joints axes are intersect, joint 2 and 3 are parallel and non co-planar but perpendicular to joint 1 ". Taking the screw kinematics model, the solution is established by using geometry relation method and basic known Paden-Kahan sub-problems. Firstly, the 1st joint angle is solved by the geometrical relationship between the robot wrist point and first three joint axes, and the rest five joint angles are solved by transforming into three Panden-Kahan subproblem 1 and one sub-problem 2, so that the complete closed form solutions for inverse kinematics are provided. The accuracy and practicality of the algorithm are testified by the results in Vgroove welding experiment.
\end{abstract}

Keywords-industry robot; screw theory; inverse kinematics; Paden-Kahan sub-problem

\section{INTRODUCTION}

Robot inverse kinematics is to calculate the angles of each joint of the robot based on the desired posture of the robot endeffector, the inverse solution is the premise and basis for the robot trajectory planning and motion control. The first step of inverse kinematics is to establish a kinematic model of the robot. At present, the kinematics model of the robot is mainly composed of the Denavit-Hartenberg(D-H) parameter method and the screw theory method. However, D-H parameter method is complex to establish the local coordinate system and requires observation and experience to establish a constraint equation with joint variables, and the analytical expression derivation process is complicated. In screw method, the inverse problem is solved by the Paden-Kahan sub problem, and avoids the singularity brought by the used of the DH coordinate method. Sariyildiz[1] uses quaternions and known Paden-Kahan subproblems to solve the common structure of the robot kinematics inverse solution. Tan[2] applied the expanded Paden-Kahan sub-problem to solve the 5-DOF robot kinematics inverse solution with special structure. Chen Weihai[3] solved the 7-DOF robot inverse kinematics problem with Paden-Kahan sub-problems and velocity level inverse solution algorithm. The disadvantage of the Paden-Kahan subproblem is that the axes of all joints of the robot must have enough intersections. In particular, the first two joint axes are intersected, which limits the generality of the algorithm.

The typical structural feature of 6-DOF welding industrial robot is that, the first 3 joints do not intersect each other, and the axes of the 2 and 3 joints are parallel, and both are perpendicular to the plane of the 1st joint axis. The structural characteristics of the first three joints make the Paden-Kahan sub-problem can be solved directly. Aiming at the problem of solving the first three joint angle, Qian[4] used the classical elimination method to solve the problem, but this method has a large amount of calculation. Chen[5] extended it to a new subproblem, but the derivation process is more complex.

Based on the screw theory, this paper presents a new method for solving the inverse kinematics of 6-DOF industry welding robot, combining the geometric relation method with Paden-Kahan subproblem. This method decomposes the whole inverse problem into three steps: First, Solve joint angle 1 by using geometric relation method. The plane constituted by axis of joint 1 and point of wrist, which is always perpendicular to the joints of 2 and 3, Thus the line connected between any point on the axis of the joint 1 and the wrist point, satisfies the vertical geometric relationship with the joints of 2 and 3, and the joint angle 1 can be solved by this feature. Second, the inverse solutions of joint angles of 2 and 3 are transformed into two Paden-Kahan sub-problems by using the parallel geometrical features of joint 2 and 3 axes. Third, By the geometric feature that 4,5 and 6 joint axes intersect at the wrist point, and the joint angles of 4 and 5 are solved by transforming into a Panden-Kahan sub-problem 2, and solution of joint angle 6 is transformed into sub-problem 1.In the above method, the inverse kinematics closed solution is given through the geometric characteristics of 6-DOF industry robot and known Paden-Kahan sub-problem. The experiment is performed at last to show the accuracy of this algorithm.

\section{Modeling AND ALGORITHM}

\section{A. Robot Kinematics Modeling}

As shown in Figure. $1, W\left\{o_{0}-x_{0} y_{0} z_{0}\right\}$ and $E\left\{o_{6}-x_{6} y_{6} z_{6}\right\}$ are set as the base and the end coordinate system respectively.

For welding robot, the welding torch should be installed at the end, $T\left\{o_{T}-x_{T} y_{T} z_{T}\right\}$ is set as the welding torch tool coordinate system. In general, It is determined of the transformation matrix ${ }_{T}^{E} T$, which is the coordinate system $T$ with respect to coordinate system $E$, and it can be precisely calibrated by the robot TCP calibration algorithm[6]. When the desired position and pose of the welding torch is given, the transformation matrix ${ }_{E}^{W} T$ which set as the end of the robot relative to the base coordinate system can be expressed as, 


$$
{ }_{E}^{W} T={ }_{T}^{W} T\left({ }_{T}^{E} T\right)^{-1}
$$

By formula(1), the problem of inverse kinematics of robot with a torch can be translated into that without welding torch, namely coordinate $E$ as the robot end for inverse solution. Therefore, the inverse problem of the robot without torch is studied as follows, and according to the representation of screw theory, ${ }_{E}^{W} T$ is recorded as $\boldsymbol{g}_{w e}(\theta)$.As shown in Figure 1.The robot configuration with each joint angle zero is defined as the reference configuration. The screw coordinate system is set up in this configuration.

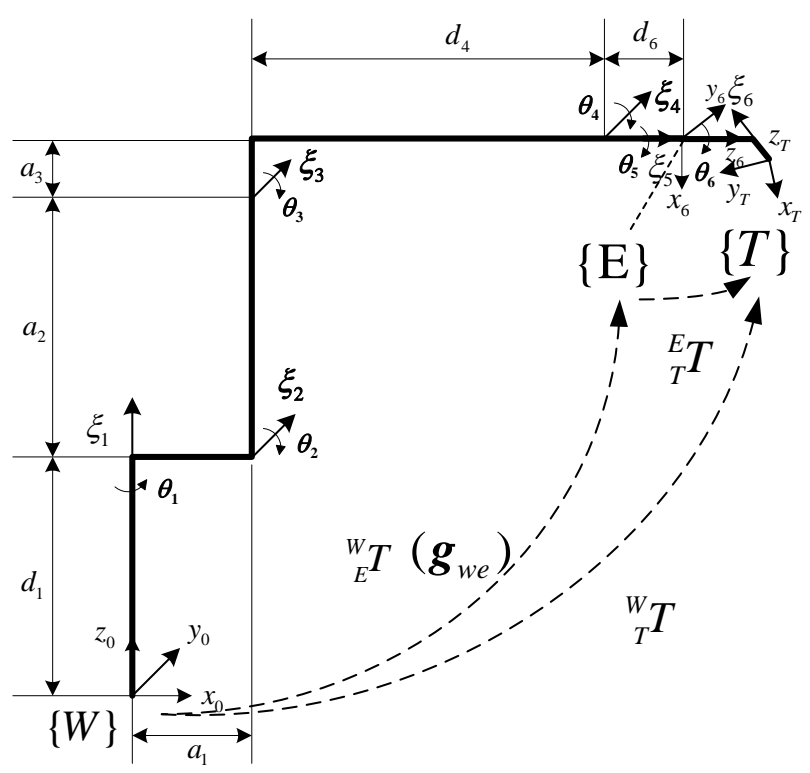

FIGURE I. SCREW COORDINATE FRAMES OF 6-DOF ROBOT

$\boldsymbol{g}_{w e}(0)$ is denoted as the transformation matrix between coordinate system $E$ and $W$, which is,

$$
\boldsymbol{g}_{w e}(\mathbf{0})=\left[\begin{array}{cccc}
0 & 0 & 1 & a_{1}+d_{4}+d_{6} \\
0 & 1 & 0 & 0 \\
-1 & 0 & 0 & a_{2}+a_{3}+d_{1} \\
0 & 0 & 0 & 1
\end{array}\right]
$$

The screw coordinates of each joint are:

$$
\begin{aligned}
& \xi_{1}=[0,0,0,0,0,1], \\
& \xi_{2}=\left[-d_{1}, 0, a_{1}, 0,0,1\right], \\
& \xi_{3}=\left[-a_{2}-d_{1}, 0, a_{1}, 0,1,0\right], \\
& \xi_{4}=\left[0, a_{2}+a_{3}+d_{1}, 0,1,0,0\right], \\
& \xi_{5}=\left[-a_{2}-a_{3}-d_{1}, 0, a_{1}+d_{4}, 0,1,0\right], \\
& \xi_{6}=\left[0, a_{2}+a_{3}+d_{1}, 0,1,0,0\right] .
\end{aligned}
$$

According to the exponential product formula of forward kinematics for robot of $n$ DOF, forward kinematics solution of robot is denoted as $\boldsymbol{g}_{w e}(\boldsymbol{\theta})$, which is,

$$
\boldsymbol{g}_{w e}(\boldsymbol{\theta})=\prod_{i=1}^{n} e^{\hat{\varepsilon}_{i} \theta_{i}} \boldsymbol{g}_{w e}(0)
$$

\section{B. Robot Inverse Kinematics Algorithm}

As shown in Figure.2, suppose $\boldsymbol{\xi}_{1}, \boldsymbol{\xi}_{2}$ and $\boldsymbol{\xi}_{3}$ as three zero pitch, mutually disjoint unit motion screw, in which $\xi_{2}$ is parallel to $\xi_{3}, \xi_{2}$ and $\xi_{3}$ are all different plane with $\boldsymbol{\xi}_{1}$, which in the plane $\boldsymbol{M}$ rotated by $\boldsymbol{\xi}_{2}$ and $\boldsymbol{\xi}_{3}$, In the initial configuration, Point $\boldsymbol{p}_{\mathrm{w} 4}$ rotates $\theta_{3}$ to $\boldsymbol{p}_{\mathrm{w} 3}$ around the axis of $\boldsymbol{\xi}_{3}$, then rotates $\theta_{2}$ to $\boldsymbol{p}_{\mathrm{w} 2}$ around the axis of $\boldsymbol{\xi}_{2}$, finally, rotates $\theta_{1}$ to $\boldsymbol{p}_{\mathrm{w} 1}$ around the axis of $\boldsymbol{\xi}_{1}$.

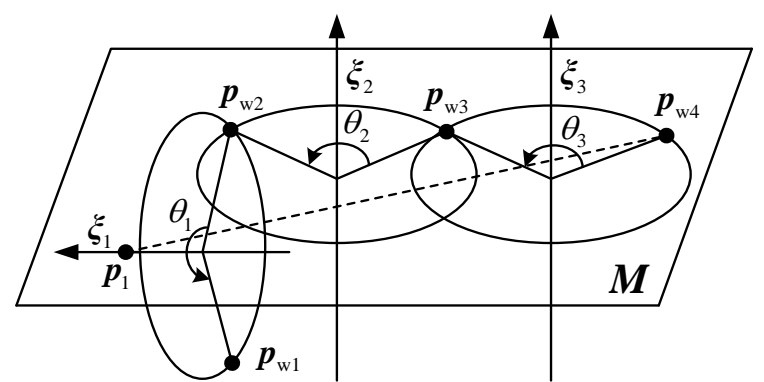

FIGURE II. INVERSE KINEMATICS SUB-PROBLEM FOR THE FIRST THREE JOINTS

Then the above sub-problem satisfies the following screw motion formula:

$$
e^{\hat{\xi}_{1} \theta_{1}} e^{\hat{\xi}_{2} \theta_{2}} e^{\hat{\xi}_{3} \theta_{3}} \boldsymbol{p}_{\mathrm{w} 4}=\boldsymbol{p}_{\mathrm{w} 1}
$$

When $\boldsymbol{p}_{\mathrm{w} 1}, \boldsymbol{p}_{\mathrm{w} 4}$ are given, $\theta_{1}, \theta_{2}$ and $\theta_{3}$ can be solved.

1) Solve the joint angle $\theta_{1}$

The last three joints of 4,5 , and 6 intersect at one point, namely the robot wrist center point, set as $\boldsymbol{p}_{\mathrm{w} 4}$, then:

$$
\boldsymbol{p}_{\mathrm{w} 4}=\boldsymbol{p}_{5}=\left\{a_{1}+d_{4}, 0, d_{1}+a_{2}+a_{3}\right\}
$$

By the Formula (3), it is can be obtained that,

$$
e^{\hat{\xi}_{1} \theta_{1}} e^{\hat{\xi}_{2} \theta_{2}} \cdots e^{\hat{\xi}_{6} \theta_{6}}=g_{s t}(\theta) g_{s t}(0)^{-1}=g_{1}
$$

Both sides of Formula(5) are right multiplied by $\boldsymbol{p}_{\mathrm{w} 4}$, which locates on the axis of $\xi_{4}, \xi_{5}$ and $\xi_{6}$, According to the principle of rigid body movement that "the position remains unchanged when the point on the revolution axis", then

$$
e^{\hat{\xi}_{1} \theta_{1}} e^{\hat{\xi}_{2} \theta_{2}} e^{\hat{\xi}_{3} \theta_{3}} \boldsymbol{p}_{\mathrm{w} 4}=\boldsymbol{g}_{1} \boldsymbol{p}_{\mathrm{w} 4}=\boldsymbol{p}_{\mathrm{w} 1}
$$


As shown in Figure.2, for $\boldsymbol{\xi}_{1}$ and $\boldsymbol{p}_{\mathrm{w} 4}$ are all located in the Plane $\boldsymbol{M}$, then the line $\boldsymbol{p}_{1} \boldsymbol{p}_{\mathrm{w} 4}$ is perpendicular to $\boldsymbol{\xi}_{2}$. As shown in Figure.3, suppose robot rotates angle $\theta_{1}$ around $\xi_{1}$.

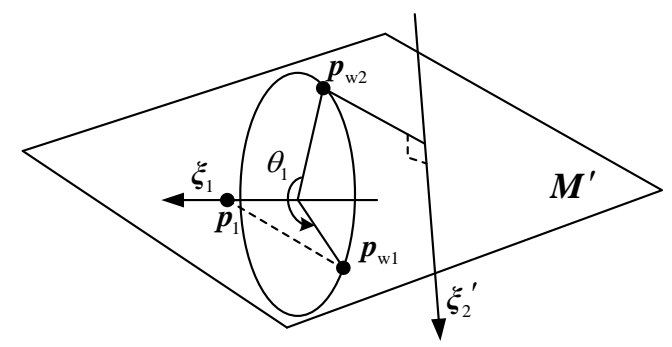

FIGURE III. THE SCREW COORDINATE FRAMES OF THE FIRST TWO

$$
\text { JOINTS AFTER ROTATION OF } \theta_{1}
$$

Then $\boldsymbol{p}_{\mathrm{w} 4}$ is rotated to $\boldsymbol{p}_{\mathrm{w} 1}$ through $\boldsymbol{p}_{\mathrm{w} 2}$ and $\boldsymbol{p}_{\mathrm{w} 3}, \boldsymbol{\xi}_{2}$ is rotated to $\xi_{2}^{\prime}$, and Plane $\boldsymbol{M}$ is become to $\boldsymbol{M}^{\prime}$, position of $\boldsymbol{p}_{1}$ remains constant, $\boldsymbol{p}_{1} \boldsymbol{p}_{\mathrm{w} 1}$ is located in Plane $\boldsymbol{M}^{\prime}$, therefore, $\boldsymbol{p}_{1} \boldsymbol{p}_{\mathrm{w} 1}$ is perpendicular to $\boldsymbol{\xi}_{2}^{\prime}$.

Set $\boldsymbol{\omega}_{2}^{\prime}$ is the corresponding rotation vector for $\xi_{2}^{\prime}$, then $\boldsymbol{\omega}_{2}^{\prime}=e^{\hat{\omega}_{1} \theta_{1}} \boldsymbol{\omega}_{2}$, it is calculated by formula of Rodrigues, then

$$
\boldsymbol{\omega}_{2}^{\prime}=\left[-\sin \left(\theta_{1}\right), \cos \left(\theta_{1}\right), 0\right]
$$

$\boldsymbol{p}_{\mathrm{w} 1}$ is derived from the formula(6), suppose $\boldsymbol{p}_{\mathrm{w} 1}=\left[p_{\mathrm{w} 1 x}, p_{\mathrm{w} 1 y}, p_{\mathrm{w} 1 z}\right]^{\mathrm{T}}$, from $\boldsymbol{p}_{1} \boldsymbol{p}_{\mathrm{w} 1} \perp \boldsymbol{\omega}_{2}^{\prime}$, then

$$
\left(\omega_{2}^{\prime}\right)^{T} \cdot\left(p_{1}-p_{\mathrm{w} 1}\right)=0
$$

From formula( $(8), \theta_{1}$ can be solved by

$$
\theta_{1}=\arctan \left( \pm p_{\mathrm{w} 1 x}, \pm p_{\mathrm{w} 1 y}\right)
$$

2) Solve the joint angle $\theta_{2}$ and $\theta_{3}$

As shown in Figure.2, the joints of 2 and 3 constitute the screw motion of two paralled joints, and the projection of the screw motion trajectory of $\boldsymbol{p}_{\mathrm{w} 4}$ in the plane $\boldsymbol{M}$ is shown in Figure.3, where $\boldsymbol{p}_{2}$ and $\boldsymbol{p}_{3}$ are the centers of rotation.

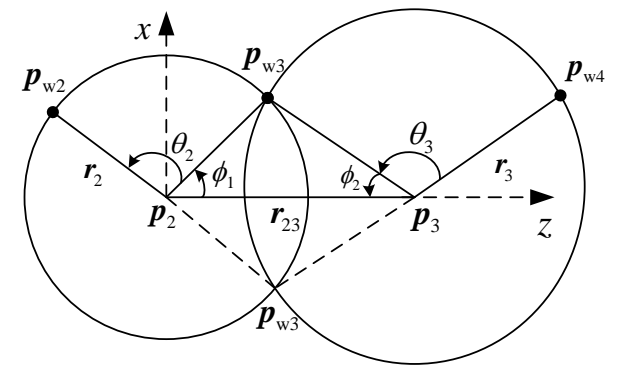

FIGURE IV. SCREW MOTION OF TWO PARALLEL JOINTS
The above can be described as inverse kinematic subproblem of two parallel joints. $\boldsymbol{p}_{\mathrm{w} 4}$ rotates $\theta_{3}$ to $\boldsymbol{p}_{\mathrm{w} 3}$ around $\boldsymbol{\xi}_{3}$, then rotates $\theta_{2}$ to $\boldsymbol{p}_{\mathrm{w} 2}$ around $\boldsymbol{\xi}_{2}$, if given $\boldsymbol{p}_{\mathrm{w} 2}$ and $\boldsymbol{p}_{\mathrm{w} 4}$, from formula $e^{\hat{\xi}_{2} \theta_{2}} e^{\hat{\xi}_{3} \theta_{3}} \boldsymbol{p}_{\mathrm{w} 4}=\boldsymbol{p}_{\mathrm{w} 2}, \theta_{2}$ and $\theta_{3}$ can be solved.

First solve $\boldsymbol{p}_{\mathrm{w} 2}$ according to the its screw motion, then,

$$
\boldsymbol{p}_{\mathrm{w} 2}=e^{-\hat{\xi}_{1} \theta_{1}} \cdot \boldsymbol{p}_{\mathrm{w} 1}
$$

If $\boldsymbol{p}_{\mathrm{w} 3}$ is solved, the following formula can be given,

$$
\left\{\begin{array}{l}
e^{-\hat{\xi}_{2} \theta_{2}} \cdot \boldsymbol{p}_{\mathrm{w} 2}=\boldsymbol{p}_{\mathrm{w} 3} \\
e^{\hat{\xi}_{3} \theta_{3}} \cdot \boldsymbol{p}_{\mathrm{w} 4}=\boldsymbol{p}_{\mathrm{w} 3}
\end{array}\right.
$$

Equation(11) is actually two Panden-Kahan sub-problem 1, so the key issue is to solve $\boldsymbol{p}_{\mathrm{w} 3}$. It is illustrated by Figure.4, $r_{2}=\left\|\boldsymbol{p}_{2}-\boldsymbol{p}_{\mathrm{w} 2}\right\|, r_{3}=\left\|\boldsymbol{p}_{3}-\boldsymbol{p}_{w 4}\right\|, r_{23}=\left\|\boldsymbol{p}_{2}-\boldsymbol{p}_{3}\right\|$, in $\Delta \boldsymbol{p}_{\mathrm{w} 3} \boldsymbol{p}_{2} \boldsymbol{p}_{3}$, Suppose the angle between $\boldsymbol{p}_{\mathrm{w} 3} \boldsymbol{p}_{2}$ and $\boldsymbol{p}_{2} \boldsymbol{p}_{3}$ as $\phi_{1}$, from the cosine theorem, then

$$
r_{2}^{2}+r_{23}{ }^{2}-2 r_{2} \cdot r_{23} \cos \phi_{1}=r_{3}^{2}
$$

$\boldsymbol{p}_{2}, \boldsymbol{p}_{3}, \boldsymbol{p}_{\mathrm{w} 2}$ and $\boldsymbol{p}_{\mathrm{w} 4}$ are substituted into formula(12), then

$$
\phi_{1}=\operatorname{acos}\left(\frac{a_{2}{ }^{2}-a_{3}{ }^{2}-d_{4}{ }^{2}+\left(q_{\mathrm{w} 2 \mathrm{x}}-a_{1}\right)^{2}+\left(q_{z}-d_{1}\right)^{2}}{2 a_{2} \cdot \sqrt{\left(q_{\mathrm{w} 2 \mathrm{x}}-a_{1}\right)^{2}+\left(q_{z}-d_{1}\right)^{2}}}\right)
$$

In the reference configuration, plane $\boldsymbol{M}$ within points of $\boldsymbol{p}_{\mathrm{w} 2}, \boldsymbol{p}_{\mathrm{w} 3}$ and $\boldsymbol{p}_{\mathrm{w} 4}$ is coincides with the $x z$ plane of the base coordinate system $W$, suppose $\boldsymbol{p}_{\mathrm{w} 3}=\left[p_{\mathrm{w} 3 x}, p_{\mathrm{w} 3 y}, p_{\mathrm{w} 3 z}\right]^{\mathrm{T}}$, then

$$
\left\{\begin{array}{l}
p_{\mathrm{w} 3 x}=p_{\mathrm{w} 2 x} \pm r_{2} \cos \left(\phi_{1}\right) \\
p_{\mathrm{w} 3 y}=0 \\
p_{\mathrm{w} 3 z}=p_{\mathrm{w} 2 \mathrm{z}}+r_{2} \sin \left(\phi_{1}\right)
\end{array}\right.
$$

Vectors are defined as follows,

$$
\begin{cases}u=p_{\mathrm{w} 2}-p_{2}, & \boldsymbol{v}=\boldsymbol{p}_{\mathrm{w} 4}-\boldsymbol{p}_{3} \\ \boldsymbol{p}_{23}=\boldsymbol{p}_{3}-\boldsymbol{p}_{2}, & \boldsymbol{p}_{32}=\boldsymbol{p}_{2}-\boldsymbol{p}_{3}\end{cases}
$$

By formula(11), then

$$
\left\{\begin{array}{l}
\theta_{2}=\arctan \left(\omega_{2}^{T}\left(\boldsymbol{p}_{23} \times \boldsymbol{u}\right), \boldsymbol{p}_{23}^{T} \boldsymbol{u}\right) \\
\theta_{3}=\arctan \left(\omega_{3}^{T}\left(\boldsymbol{v} \times \boldsymbol{p}_{32}\right), \boldsymbol{v}^{T} \boldsymbol{p}_{32}\right)
\end{array}\right.
$$


3) Solve the joint angle $\theta_{4}, \theta_{5}$ and $\theta_{6}$

After solving the above three joint angles, the inverse solution of the last 3 joints can be solved by using the known Paden-Kahan sub-problem. By formula(5), then

$$
e^{\hat{\xi}_{4} \theta_{4}} e^{\hat{\xi}_{5} \theta_{5}} e^{\hat{\xi}_{6} \theta_{6}}=e^{-\hat{\xi}_{1} \theta_{1}} e^{-\hat{\xi}_{2} \theta_{2}} e^{-\hat{\xi}_{3} \theta_{3}} g_{1}=g_{2}
$$

Take point $\boldsymbol{p}_{\mathrm{w} 6}$ on axis of $\boldsymbol{\xi}_{6}$ but not on $\boldsymbol{\xi}_{4}$ and $\boldsymbol{\xi}_{5}$, Both sides of formula(17) are right multiplied by $\boldsymbol{p}_{\mathrm{w} 6}$, then

$$
e^{\hat{\xi}_{4} \theta_{4}} e^{\hat{\xi}_{5} \theta_{5}} \boldsymbol{p}_{\mathrm{w} 6}=\boldsymbol{g}_{2} \boldsymbol{p}_{\mathrm{w} 6}=\boldsymbol{q}_{6}=\left[q_{6 x}, q_{6 y}, q_{6 z}\right]^{\mathrm{T}}
$$

By Paden-Kahan sub-problem 2, $\theta_{4}, \theta_{5}$ can be solved as

$$
\left\{\begin{array}{l}
\theta_{4}=-\arctan \left( \pm\left(q_{6 z}-a_{2}-a_{3}-d_{1}\right), \pm q_{6 y}\right) \\
\theta_{5}=\arctan \left(q_{6 x}-a_{1}-d_{4}, \mp \sqrt{d_{6}^{2}-\left(a_{1}+d_{4}-q_{6 x}\right)^{2}}\right)
\end{array}\right.
$$

By formula(5), then

$$
e^{\hat{\xi}_{6} \theta_{6}}=e^{-\hat{\xi}_{5} \theta_{5}} e^{-\hat{\xi}_{4} \theta_{4}} e^{-\hat{\xi}_{1} \theta_{1}} e^{-\hat{\xi}_{2} \theta_{2}} e^{-\hat{\xi}_{3} \theta_{3}} \boldsymbol{g}_{1}=\boldsymbol{g}_{3}
$$

Take $\boldsymbol{p}_{t}$ as any point not on the sixth axis, such as $\boldsymbol{p}_{t}=\boldsymbol{p}_{1}$, Both sides of formula(20) are right multiplied by $\boldsymbol{p}_{t}$, then

$$
e^{\hat{\varepsilon}_{6} \theta_{6}} \boldsymbol{p}_{t}=\boldsymbol{g}_{3} \boldsymbol{p}_{t}=\left[\begin{array}{lll}
q_{t x} & q_{t y} & q_{t z}
\end{array}\right]^{\mathrm{T}}
$$

By Paden-Kahan sub-problem 1, we can get

$$
\theta_{6}=\arctan \left(a_{2}+a_{3}+d_{1}-q_{t z}, q_{t y}\right)
$$

So far, all the closed solutions for 6-DOF inverse kinematics of robot have been obtained.

\section{ALGORITHM APPLICATION IN WELDING EXPERIMENT}

In order to verify the correctness of the inverse kinematics, a V-groove butt-jointed seam welding experiment is performed, and robot ABB IRB1410 is taken as welding robot, As shown in Figure.1, the link parameters of the robot are as follows(unit: $\mathrm{mm}$ ):

$$
d_{1}=475, a_{1}=150, a_{2}=600, a_{3}=120, d_{4}=720, d_{6}=85 .
$$

The workpiece is composed of two Q345 low carbon steel, the thickness of the plate is $50 \mathrm{~mm}$, and the angle of the groove is , The diameter of the welding wire is $1.0 \mathrm{~mm}$, the deposition coefficient is 0.9 , the wire feeding speed of the backing welding is $2.5 \mathrm{~mm} / \mathrm{s}$, the filler welding and the cover welding are both $7.5 \mathrm{~mm} / \mathrm{s}$. The experimental steps are as follows:

\section{1) Determine the transformation matrix ${ }_{T}^{E} T$}

${ }_{T}^{E} T$ is calibrated by the robot TCP calibration, which is,

$$
{ }_{T}^{E} T=\left[\begin{array}{cccc}
0.7038 & 0.0299 & -0.7098 & -3.119 \\
0.0151 & -0.9995 & -0.0271 & 1.102 \\
-0.7103 & 0.0083 & -0.7039 & 325.494 \\
0 & 0 & 0 & 1
\end{array}\right]
$$

2) Determine the position and pose matrix ${ }_{E}^{W} T$

${ }_{E}^{W} T$ is determined by the expected planning points along welding seam. Take the starting point of V-groove seam in bottom welding as example, which is,

$$
{ }_{E}^{W} T=\left[\begin{array}{cccc}
-0.6873 & -0.0400 & 0.7253 & 940.044 \\
-0.0443 & 0.9989 & 0.0131 & 52.026 \\
-0.7250 & -0.2232 & -0.6883 & 743.756 \\
0 & 0 & 0 & 1
\end{array}\right]
$$

3) Determine the optimum solution of robot joint angles

The inverse kinematics algorithm is used to solve the robot joint angles according to ${ }_{E}^{W} T$, and a total set of eight solutions is obtained, as shown in Table 1.

TABLE I. EIGHT SOLUTIONS OF INVERSE KINEMATICS

\begin{tabular}{|c|c|c|c|c|c|c|}
\hline No. & $\theta_{1} /\left(^{\circ}\right)$ & $\theta_{2} /\left(^{\circ}\right)$ & $\theta_{3} /\left(^{\circ}\right)$ & $\theta_{4} /\left(^{\circ}\right)$ & $\theta_{5} /\left(^{\circ}\right)$ & $\theta_{6} /\left(^{\circ}\right)$ \\
\hline 1 & 3.32 & 126.74 & 172.67 & 178.29 & -104.10 & 179.32 \\
\hline 2 & 3.32 & 126.74 & 172.67 & -1.71 & 104.10 & -0.68 \\
\hline 3 & 3.32 & 4.96 & 26.25 & 172.28 & -12.42 & -172.72 \\
\hline 4 & 3.32 & 4.96 & 26.25 & -7.72 & 12.42 & 7.285 \\
\hline 5 & -176.68 & -32.48 & -152.25 & -2.64 & -38.83 & -178.20 \\
\hline 6 & -176.68 & -32.48 & -152.25 & 177.36 & 38.83 & 1.80 \\
\hline 7 & -176.68 & -112.26 & -8.83 & -1.70 & -102.42 & 179.37 \\
\hline 8 & -176.68 & -112.26 & -8.83 & 178.30 & 102.42 & -0.63 \\
\hline
\end{tabular}

In practical application, an optimal solutions can be selected by applying the optimal conditions such as the shortest path principle. In this example, the fourth group solution is optimal.

\section{4) Offline programming and Experiment}

Four layers with ten passes are planned in off-line programming, which is shown in Figure 5 as follows:

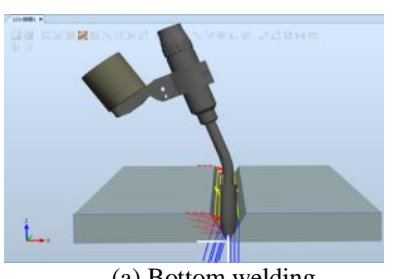

(a) Bottom welding

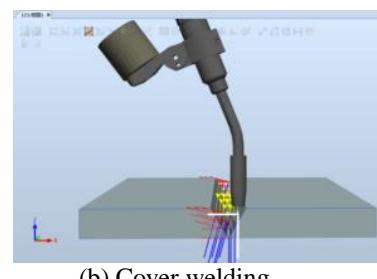

(b) Cover welding
FIGURE V. OFF-LINE PROGRAM PLANNING OF WELDING

The cooresponding program is generated and the experiment is performed as shown in Figure 6, the results show that the welding gun can tracking the weld seam accurately. 


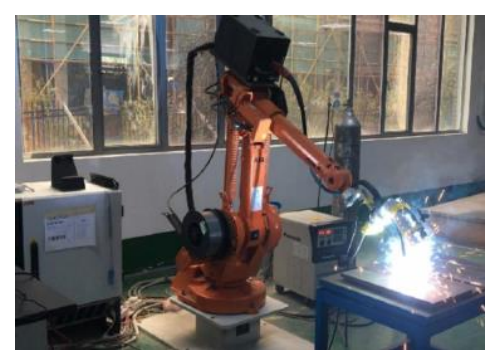

FIGURE VI. THE EXPERIMENT OF MULTI-LAYER AND MULTI-PASS WELDING

\section{CONCLUSIONS}

This paper aims at the inverse kinematics for 6-DOF industrial robots. Based on screw theory, a new method is proposed for inverse solution of kinematics according to its structural characteristics. Firstly, the joint angle 1 is solved by the geometrical relationship between the robot wrist point and first three joint axes, and the rest angles are decomposed into several known Panden-Kahan sub-problems. The accuracy and reliability of the method is proved by the results in welding experiment. It has the value of promotion and application in the actual motion control of 6-DOF industrial robots.

\section{ACKNOWLEDGMENT}

We are grateful to the reviewers and the editors for their constructive suggestions. The research of this paper is supported by the National Natural Science Funds of China (Granted No. 61703200), Scientific Research Fund Project of Nanjing Insititute of Technology (Granted No. PTKJ201606), Scientific Research Fund Project of Nanjing Insititute of Technology (Granted No. PTKJ201701), Scientific Research Fund for the introduction talent of Nanjing Insititute of Technology (Granted No. YKJ201741), and sponsored by Qing Lan Project.

\section{REFERENCES}

[1] SARIYILDIZ E, TEMELTAS H. Solution of inverse kinematic problem for serial robot using quaternions[C]. IEEE Inter Conf. on Mechatronics and Automation. Changchun: IEEE, 2009: 26-31.

[2] Tan Yue-sheng, Xiao Ai-ping. Extension of the second paden-kahan sub problem and its' application in the inverse kinematics of a manipulator[C]. IEEE Inter Conf. on Robotics, Automation and Mechatronics. Chengdu: IEEE, 2008: 379-381

[3] Chen wei-hai, Chen Quan-zhu, Zhang Jian-bin. Inverse kinematic analysis for cable-driven humanoid-arm manipulator[J]. Chinese Journal of Mechanical Engineering, 2007, 43(4):12-20.

[4] [10] Qian Dong-hai, Wang Xin-feng, Zhao Wei. Algorithm for the inverse kinematic calculation of 6-DOF robots based on screw theory and Paden-Kahan sub-problems[J]. Chinese Journal of Mechanical Engineering, 2009, 45(9):72-76.

[5] [11] Chen Qing-cheng, Zhu Shi-qiang, Wang Xuan-yin. Inverse kinematics sub-problem solution algorithm for serial robot based on screw theory[J]. Journal of Zhejiang University (Engineering Science), 2014, 48(1): 8-14.

[6] [12] Hong Lei, JI Bao-jian, SHEN Jian, etc. Research on an algorithm for industrial robot TCP calibration[J]. Journal of Machine Design, 2017, 34(3): $81-85$ 